\title{
Prevalence of Group A b-Hemolytic Streptococcus Among Children with Tonsillopharyngitis in Kyrgyzstan: The Difficulty of Diagnostics and Therapy
}

\author{
Nazgul A. Omurzakova ${ }^{*}, 1,3$, Yoshihisa Yamano ${ }^{1}$, Guli M. Saatova ${ }^{5}$, Mayramkan S. Alybaeva ${ }^{6}$, \\ Kusuki Nishioka ${ }^{1}$ and Toshihiro Nakajima ${ }^{*, 2,4}$ \\ ${ }^{I}$ Institute of Medical Science, St. Marianna University, Kawasaki, Japan \\ ${ }^{2}$ Choju Medical Institute, Fukushimura Hospital, Toyohashi, Japan \\ ${ }^{3}$ National Center of Cardiology and Internal Medicine of Health Service Ministry of the Kyrgyz Republic, Bishkek, \\ Kyrgyzstan \\ ${ }^{4}$ Center for Rheumatology and Joint diseases, Misato Marine Hospital, Kochi, Japan \\ ${ }^{5}$ National Center of Pediatrics and Child Surgery Ministry of Health of the Kyrgyz Republic, Bishkek, Kyrgyzstan \\ ${ }^{6}$ Republican Clinical Hospital of Infectious Diseases, Bishkek, Kyrgyzstan
}

\begin{abstract}
Objective: Rheumatic fever (RF) is well known disease as a result of frequent complication of the group A bhemolytic streptococcal (GABHS) tonsillopharyngitis, have noticeably grown for the last 15 years among young generation in the Kyrgyz Republic. It is important to hold a study about the prevalence of RF and GABHS and their susceptibility to antibiotics in the Kyrgyz Republic.

Materials and Methods: We have studied 200 children with chronic tonsillopharyngitis at the National Center of Pediatrics and Child Surgery in Bishkek (the Kyrgyz Republic) from August till September 2008. 188 (48 out of them are with RF) out of total 200 children are Kyrgyz and 12 are Russian ( 2 out of them are with RF). 111 out of total are female (34 out of them are with RF) and 89 are male (17 out of them are with RF). The average age of the subjects is $10.9 \pm 6.0$ (from 3 to 17 years old). The presence of GABHS was assessed using two ways: by the rapid antigen detection test (RADT) for outcrop of streptococcus antigen in smear from mucosal surface of tonsils, and by bacterial culture analysis (BA). RADT was used to determine its specificity and sensitivity in order to recommend practitioners its further wide use. Furthermore, the discovered culture of GABHS have been investigated on susceptibility to antibiotics by disc-diffusion method.

Research Results: GABHS antigen was positive in $72(36.0 \%)$ subjects from RADT and in $80(40.0 \%)$ subjects from BA out of 200 total subjects. In the patients with RF ( $\mathrm{n}=51)$, GABHS was positive in $18(35.2 \%)$ subjects by RADT and in 24 $(47.0 \%)$ subjects by BA. In the subjects without RF ( $n=149)$, GABHS was positive in $54(36.2 \%)$ subjects by RADT and in $56(37.5 \%)$ by BA. Among 80 GABHS positive results sensitive to antibiotics were: to penicillin only $10(12.5 \%)$, to ampicillin-29 (36.2\%), to amoxicillin-36 (45.0\%), to ceftriaxon - $31(38.7 \%)$, to roxithromycin - 21 (26.2\%), to erythromycin- $19(23.7 \%)$. It is noted that $21(26.2 \%)$ GABHS positive results were absolutely resistant for all these tested antibiotics. RADT showed that its specificity is $85 \%$ as well as its sensitivity is equal to $67.5 \%$.

Conclusion: In this study the sensitivity of RADT was low; therefore, the negative results of RADT don't exclude presence of GABHS. High prevalence of GABHS antigen demonstrates not only in patients with RF, but also among healthy children (without RF) of the Kyrgyz Republic. The high prevalence of GABHS at children with RF (47.0\%), probably, presents a low sensitivity to antibiotics and irregular secondary prophylaxis. Significant presence of GABHS among healthy children (37.5\%) requires improvement of primary prevention to prevent further spread of RF and Rheumatic Heart Disease (RHD) in the country.
\end{abstract}

Keywords: Group A b-hemolytic streptococcus (GABHS), rheumatic fever (RF), rapid antigen detection test (RADT), bacterial culture analysis (BA), antibiotic-sensitivity.

*Address correspondence to these authors at the (NAO) Institute of Medical Science St. Marianna University School of Medicine, 2-16-1 Sugao, Miyamae-ku, Kawasaki, Kanagawa 216-8512, Japan; Tel: +81-44-9778111, Ext. 4113; Fax:+81-44-977-9772; E-mail: nazbunisa@gmail.com and (TN) Institute of Medical Science St. Marianna University School of Medicine, 2-16-1 Sugao, Miyamae-ku, Kawasaki, Kanagawa 216-8512, Japan; Tel: +81-44-977-8111, Ext. 4113; Fax: +81-44-977-9772;

E-mail: nakasix@n06.itscom.net

\section{INTRODUCTION}

Collapse of the Union of the Soviet Socialist Republics (USSR) in the beginning of 1990 strongly reflected in the sector of public health services of many former union republics. For the last 15 years in the Kyrgyz Republic, which is located in the Central Asia, there has been a growth of many infectious diseases and their complications, among 
which a streptococcal tonsillopharyngitis and its complication as a rheumatic fever (RF) has a special importance. The key parameter for an estimation of the significance of tonsillopharyngitis is a prevalence of RF [25, 6, 8-10]. Rheumatic fever and rheumatic heart disease (RHD) are frequent reason of mortality, including a sudden death and an early invalidity of young generations, leading to economical damages in this country. The statistics of growth of RF and RHD in Kyrgyzstan has been presented at the 13th international Congress of the APLAR (Asia Pacific League of Associations for Rheumatology) in Yokohama (Japan) in September 2008 [1]. Unfortunately, Kyrgyzstan is in the leading position on distribution of the RF among all developing countries of the world (643 cases/ 100, 000 individuals) $[1-3,5]$. While in the developed countries, including Japan, the incidence of RF/RHD 0, 8-1, 0 patients per 100000 population [2-7].

$\mathrm{RF}$ is an autoimmune disease primarily of children between ages 5 and 15 years, which develops approximately 20 days after group A b-hemolytic streptococcal (GABHS) tonsillopharyngitis and affect heart, central nervous system, joints and skin. According to the report of the US Vital Health Statistics, an acute tonsillopharyngitis causes more than 6 million policlinic visits each year by children younger than 15 years of age and an additional about 1.8 million visits by adolescents and young adults aged 15 to 24 years [8]. According to some publications, approximately $28 \%$ to $40 \%$ of the tonsillopharyngitis of bacteriological etiology are estimated to be caused by GABHS (or Streptococcus pyogenes), which is considered as the most important etiological human pathogen in the frequency of complications $[9,10]$. Tonsillopharyngitis is one of the most ordinary common infections encountered by pediatricians and family physicians in Kyrgyzstan. Unfortunately, the official statistics about prevalence of the GABHStonsillopharyngitis is not available in Kyrgyzstan. Streptococcal tonsillitis or angina and pharyngitis represent a large source of social inconveniences especially among children's and adolescent population. It can lead to a missing school days, the need of using antimicrobial drugs repeatedly, and the potential risk of suppurative and nonsuppurative complications, such as peritonsillar abscess, diffuse glomerulonephritis and rheumatic fever. Besides, the infection that remains after acute streptococcal tonsillopharyngitis is kept in many recovered throats and tonsils in a dozing condition. Such "healthy" people represent the tank of infection and pathogenic streptococcus can transfer from carriers to susceptible persons. It is a commonly known that GABHS can be transmitted easily from asymptomatic carriers as well as patients, with GABHS tonsillopharyngitis. Our joint Kyrgyz-Japanese study among children and adult population in Kyrgyzstan in 2007 have revealed that $37 \%$ subjects out of 189 ethnic Kyrgyz population are carriers of this streptococcus [11]. Considering the high growth of RF/RHD in Kyrgyzstan, it is necessary to note that accurate diagnosis of the GABHS infection is important. Since it is the only common form of the acute tonsillopharyngitis, for which antibiotic therapy is obligatory, when possible shortening the clinical course of the GABHS tonsillopharyngitis, reducing the transmission of infection, and prevention of complications, among which the acute rheumatic fever occupies a special place. Due to an important role that streptococcal infection has in Kyrgyzstan, the efforts leading to better understanding of its risk factors are necessary. This study aims to identify and compare the prevalence of Streptococcus pyogenes detected by Rapid Antigen Detection Test (RADT) and by bacterial culture analysis (BA) from the oropharynx microbial flora of children with tonsillopharyngitis among different age groups. The study also determines the susceptibility of discovered GABHS to different groups of antibiotics.

\section{MATERIALS AND METHODS}

We have studied 200 children with chronic tonsillopharyngitis at the National Center of Pediatrics and Child Surgery in Bishkek (Kyrgyzstan) from August till September 2008.

The study was held in accordance with the permission of the ethical committee on patients' rights and voluntary signed consent of the parents of children. (Patients' rights and medical ethics. Information materials for physicians, Bishkek, 1999).

Table 1. Information of the Total Subjects

\begin{tabular}{|l|r|}
\hline \multicolumn{1}{|c|}{$\mathbf{n}$} & \multicolumn{1}{|c|}{ Freguency (\%) } \\
\hline \hline Male/Female & $89 / 111$ \\
\hline Age at onset $(\mathbf{y r} \pm$ SD) & $10.9 \pm 6.0$ \\
\hline Kyrgyz & $188(94.0 \%)$ \\
\hline Russian & $12(6.0 \%)$ \\
\hline With RF & $51(25.5 \%)$ \\
\hline Without RF & $149(74.5 \%)$ \\
\hline RF-rheumatic fever. & \\
\hline
\end{tabular}

188 out of total children with chronic tonsillopharyngitis are Kyrgyz (49 out of them are with RF) and 12 are Russian ( 2 out of them are with RF) (Table 1). 111 out of total are female (34 out of them are with RF) and 89 are male (17 out of them are with RF). The average age of the subjects is $10.9 \pm 6.0$ (from 3 to 17 years old). In order to analyze the prevalence of streptococcus pyogenes and rheumatic fever in different age ranging, children were divided into 3 groups (Table 2): in the group of 3-6 years $(n=30) 7$ children with $R F$; in the group of $7-12$ years $(n=78) 22$ children with $R F$; in the group of 13-17 years $(n=92) 22$ children with RF.

Inclusions criteria: children (not younger than 3 years and older than 17 years) with chronic tonsillopharyngitis (history of persistent tonsillitis 2 and more per year). At the moment of the study many children received antibacterial therapy and others were receiving antibiotics, but it was not an exceptional criterion for us.

Exclusion criteria: previous tonsillectomy; congenital or acquired immunodeficiency.

\section{METHODS OF RESEARCH}

Common clinical inspection (complaints, anamnesis and objective inspection of patient), social anamnesis, 
Table 2. The Groups in Accordance with Age Ranging

\begin{tabular}{|c|r|}
\hline \multicolumn{1}{|c|}{$\mathbf{n}$} & \multicolumn{1}{|c|}{ Frequency $(\%)$} \\
\hline \hline Children with RF & $7(23.3 \%)$ \\
\hline 3-6 years $(\mathbf{n}=\mathbf{3 0})$ & $22(28.2 \%)$ \\
\hline 7-12 years $(\mathbf{n}=\mathbf{7 8})$ & $22(23.9 \%)$ \\
\hline 13-17 years $(\mathbf{n}=\mathbf{9 2})$ & \\
\hline Children without $\mathbf{R F}$ & $23(76.7 \%)$ \\
\hline 3-6 years $(\mathbf{n}=\mathbf{3 0})$ & $56(71.8 \%)$ \\
\hline 7-12 years $(\mathbf{n}=\mathbf{7 8})$ & $70(76.1 \%)$ \\
\hline 13-17 years $(\mathbf{n}=\mathbf{9 2})$ & \\
\hline RF-rheumatic fever. & \\
\hline
\end{tabular}

anthropomorphic measurement: growth, body weight. The samples were taken since August till September 2008 and were collected from the patient's posterior pharynx and tonsil surfaces by physicians as recommended by the Infectious Disease Society of America [12]. Collected samples for the rapid detection test (RADT) QuickVue Dipstick Strep A test. (Quidel Inc. San Diego, CA, USA) were performed right at the places, according to the method provided by the manufacturer. The rapid detection test is based on the detection of the GABHS antigen through an antigen agglutination reaction. In the meanwhile, all these samples from mucosal surfaces were taken for the bacterial culture analysis (BA) and identification for GABHS. The material was immediately placed on culture medium with blood-agar by a microbiologist in all samples. The samples were taken as per the microbiologic protocol described as follows: culture dishes were immediately taken to a lab and incubated in a bacteriology furnace at $36^{\circ} \mathrm{C}$ with environment enriched in $\mathrm{CO} 2$ for 24 hours. After 24 hours, the plates were assessed regarding the colonies growth and morphology. Those samples, which did not show bacterial growth, were incubated again for 24 more hours. The samples were taken and studied with identification of bacteria according to the manual of techniques established by the WHO in the "Basic Laboratory Procedures in Clinical Bacteriology" [13, 14]. Furthermore, the discovered GABHS from smear have been investigated on susceptibility to antibiotics, which were determined by the discdiffusion method with discs: ampicillin, amoxicillin, ceftriaxon, penicillin, roxithromycin, erythromycin (Sankt-Petersburg, Russia). Estimation of results of definition of susceptibility of bacteria to antibiotics by disc-diffuse method was carried out in accordance with recommendations of the National Committee for Clinical Laboratory Standards (NCCLS) now called Clinical and Laboratory Standards Institute (CLSI) $[15,16]$. Samples taken in the National Center of Pediatrics and the Child Surgery of Ministry of Health of the Kyrgyz Republic were submitted for a culture and analysis to the Bishkek Republican Clinical Hospital of Infectious Diseases (at the clinical bacteriological laboratory of infectious diseases) in Kyrgyzstan.

\section{RESULT OF RESEARCH}

\section{GABHS Positive Subjects in Total}

To detect the GABHS antigen, we used useful for speedy and accurate diagnostics of streptococcal tonsillopharyngitis RADT, and also studied all smear from throat by bacterial culture analysis (BA) in parallel. As shown in Table 3. 72
(36.0\%) subjects were positive for GABHS antigen by RADT and $80(40.0 \%)$ were positive by BA out of total 200 subjects (18 positive results from 72 positive by RADT were negative by $\mathrm{BA}$, and 26 negative results by RADT were positive by BA). In the patients with RF $(n=51)$, GABHS was positive in 18 $(35.2 \%)$ subjects by RADT and in $24(47.0 \%)$ subjects by BA. Out of 149 children without RF, 54 (36.2\%) subjects by RADT and $56(37.5 \%)$ subjects by BA had positive results.

Table 3. Prevalence of GABHS in Subjects

\begin{tabular}{|c|c|}
\hline $\mathbf{n}$ & Frequency (\%) \\
\hline \multicolumn{2}{|c|}{ GABHS positive by RADT } \\
\hline Total $(n=200)$ & $72(36.0 \%)$ \\
\hline With RF (n=51) & $18(35.2 \%$ \\
\hline Without RF (n=149) & $54(36.2 \%$ \\
\hline \multicolumn{2}{|l|}{ GABHS positive by BA } \\
\hline Total $(n=200)$ & $80(40.0 \%$ \\
\hline With RF $(n=51)$ & $24(47.0 \%$ \\
\hline Without RF $(n=149)$ & $56(37.5 \%$ \\
\hline
\end{tabular}

\section{Prevalence of GABHS in Children with RF}

We analyzed each group more in detail (in the children with and without RF) separately. In Table 4 out of total 51 children with RF, 18 (35.2\%) subjects by RADT and 24 (47.0\%) by BA were positive for GABHS (3 subjects from 18 positive subjects by RADT were negative by BA and 9 negative subjects by RADT had positive results by BA). Among 2 Russian children in this group nobody has positive results by both methods. Thus, the high prevalence of GABHS among total children by RADT and by BA is due to the large number of carriers of this pathogenic microbe among Kyrgyz children with RF.

Table 4. Prevalence of GABHS in Children with RF

\begin{tabular}{|c|c|}
\hline $\mathbf{n}$ & Frequency (\%) \\
\hline \multicolumn{2}{|c|}{ GABHS positive by RADT } \\
\hline Total $(n=51)$ & $18(35.2 \%)$ \\
\hline Kyrgyz $(n=49)$ & $18(36.7 \%)$ \\
\hline Russian (n=0) & - \\
\hline \multicolumn{2}{|c|}{ GABHS positive by BA } \\
\hline Total $(n=51)$ & $24(47.0 \%)$ \\
\hline Kyrgyz $(n=49)$ & $24(48.9 \%)$ \\
\hline Russian (n=0) & - \\
\hline
\end{tabular}

\section{Prevalence of GABHS in Children without RF}

In group without RF (Table 5), 54 (36.2\%) by RADT and $56(37.5 \%)$ by BA out of 149 total subjects were positive for GABHS (15 from 54 positive by RADT were negative by 
BA and 17 negative by RADT were positive by BA). We inspected only 10 Russian children without RF where 3 $(30.0 \%)$ by RADT and $2(20.0 \%)$ by BA out of them had positive results (all 3 children positive by RADT were negative by BA, and 2 subjects negative by RADT were positive by BA). Among 139 Kyrgyz children without RF there were $51(36.6 \%)$ positive cases by RADT and 54 $(38.8 \%)$ by BA (12 positive subjects by RADT were negative by BA and 15 positive subjects by BA were negative by RADT). Thus, the prevalence of GABHS in Kyrgyz children without RF was higher than in Russian children.

Table 5. Prevalence of GABHS in Children without RF

\begin{tabular}{|c|c|}
\hline $\mathbf{n}$ & Frequency (\%) \\
\hline \multicolumn{2}{|c|}{ GABHS positive by RADT } \\
\hline Total $(n=149)$ & $54(36.2 \%)$ \\
\hline Kyrgyz $(n=139)$ & $51(36.6 \%)$ \\
\hline Russian (n=10) & $3(30.0 \%)$ \\
\hline \multicolumn{2}{|c|}{ GABHS positive by BA } \\
\hline Total $(n=149)$ & $56(37.5 \%)$ \\
\hline Kyrgyz $(n=139)$ & $54(38.8 \%)$ \\
\hline $\operatorname{Russian}(n=10)$ & $2(20.0 \%)$ \\
\hline
\end{tabular}

\section{Prevalence of GABHS Among Female and Male Group}

Comparison of the prevalence of GABHS in female and male group in a separate way you can see in Fig. (1). In total children with RF $(n=51)$, out of 34 female $11(32.3 \%)$ subjects by RADT and $17(50.0 \%)$ subjects by BA had positive results. Out of 17 male with RF 7 (41.1\%) subjects by RADT and $7(41.1 \%)$ by BA were positive for GABHS. At children without RF $(n=149)$, out of 77 female 36 (46.7\%) subjects by RADT and 35 (45.4\%) subjects by BA had positive results. And out of 72 male without RF 18 $(25.0 \%)$ by RADT and $21(29.1 \%)$ subjects by BA were positive for GABHS.

\section{Comparison of the Prevalence of GABHS in Groups According with Ages Ranging}

The prevalence of GABHS depending on age's category has showed in Fig. (2). In group 3-6 years $(n=30)$ (Table 2) from children with RF $(n=7)$ there was $1(14.2 \%)$ positive by RADT and $4(57.1 \%)$ positive by BA and from children without RF $(\mathrm{n}=23), 5(21.7 \%)$ positive by RADT and 6 $(26.0 \%)$ positive by BA. In group $7-12$ years $(n=78)$ out of 22 subjects with RF $13(59.0 \%)$ by RADT and $11(50.0 \%)$ by $\mathrm{BA}$ had GABHS, out of 56 subjects without RF 12 $(21.4 \%)$ positive by RADT and $17(30.3 \%)$ positive by BA. In group 13-17 years $(n=92)$ out of 22 children with RF 4 $(18.1 \%)$ by RADT and $9(40.9 \%)$ by BA were positive for GABHS, and out of 70 children without RF there were 20 (28.5\%) by RADT and $29(41.4 \%)$ by BA positive subjects.

\section{Study of GABHS for Susceptibility to Antibiotics}

From total 200 subjects there were detected some pathogenic bacteria's by BA (Fig. 3A). Thus $36(18.0 \%)$ children were carriers of Streptococcus pneumoniae (S. pneumoniae) and 6 subjects out of them had also positive results for GABHS (Streptococcus pyogenes). 26 (13.0\%) subjects had Staphylococcus aureus (S. aureus) and 4 subjects out of them were positive for GABHS. And 9 (4.5\%) subjects had Pseudomonas aeruginosa (P. aeruginosa); it is conditionally pathogenic microorganism, which can be rarely presented at a nasopharynx. The data

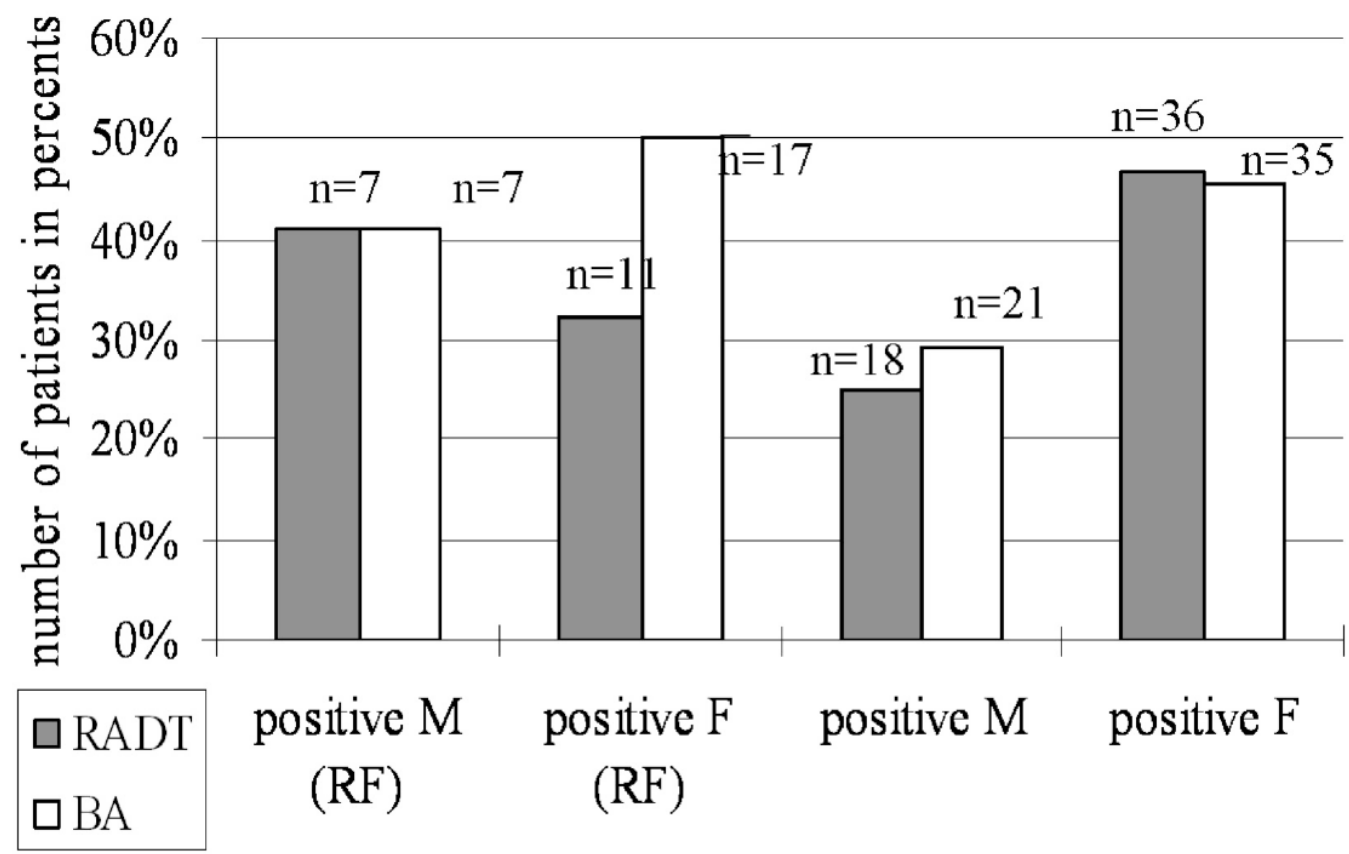

Fig. (1). Comparison of prevalence GABHS detected by rapid antigen detection test (RADT) and by bacterial culture analysis (BA) in female (F) and male (M) group among children with- and without rheumatic fever (RF). 


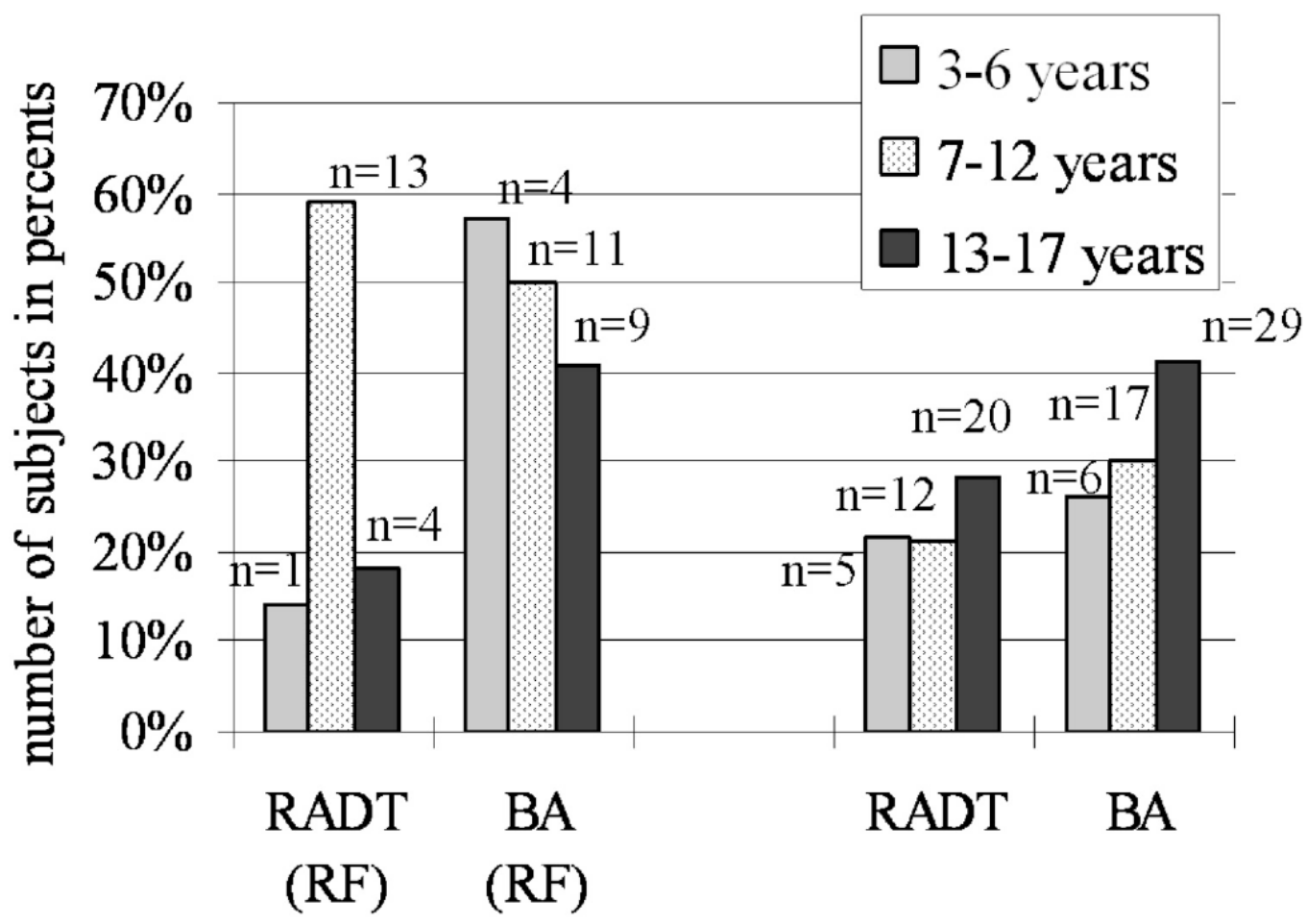

Fig. (2). Prevalence of GABHS in groups according with ages ranging among children with- and without RF.

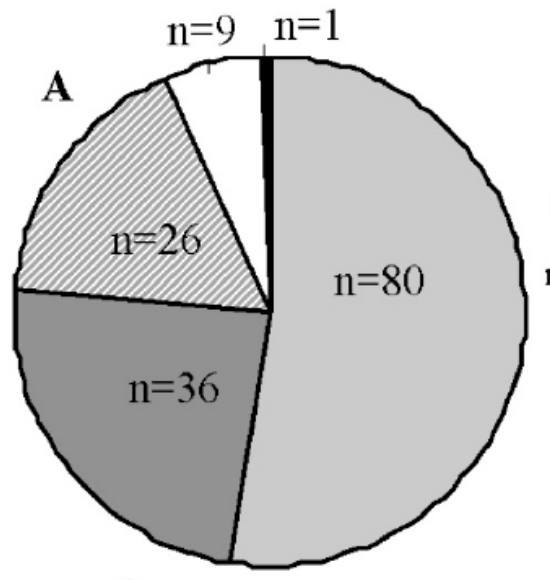

S. pyogenes

$\square$ S. pneumoniae

๑ S. aureus

$\square \mathrm{P}$. acruginosa

S. viridans

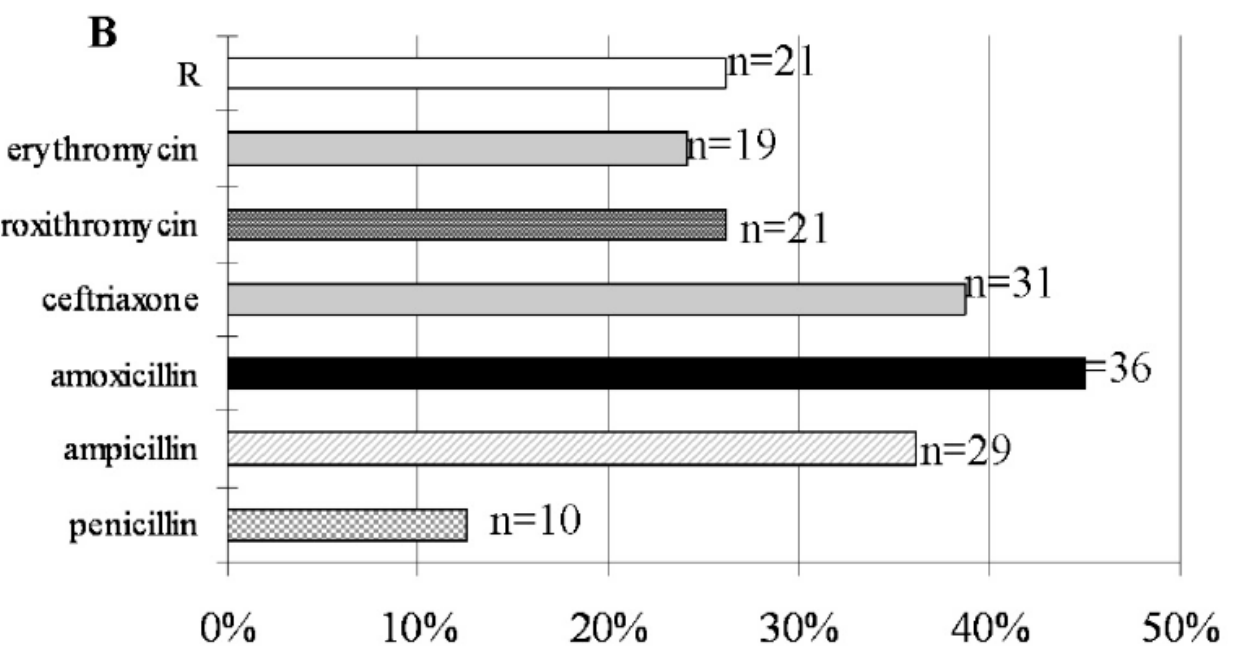

number of subjects in percents

Fig. (3). (A) Pathogenic bacteria's detected by bacterial culture analysis (BA) from 200 total subjects. (B) GABHS detected by BA were tested for sensitivity to antibiotics. 21 samples out of 80 GABHS positive subjects are absolutely resistant (R) to all these antibiotics.

results of GABHS from smear have been investigated on sensitivity and resistance to antibiotics (Fig. 3B). Among 80 GABHS positive samples detected by BA sensitive to antibiotics were: to penicillin only $10(12.5 \%)$, to ampicillin$29(36.2 \%)$, to amoxicillin-36 (45.0\%), to ceftriaxon - 31 $(38.7 \%)$, to roxithromycin - $21(26.2 \%)$, to erythromycin- 19 $(23.7 \%)$. It is noted that $21(26.2 \%)$ GABHS positive samples were absolutely resistant for all these antibiotics.

\section{RESULTS OF RADT AND BA FOR COMPARE}

In our study population as you can see from Table 6, sensitivity of RADT was $54 / 80$ or $67.5 \%$ and specificity was $102 / 120$ or $85 \%$.

\section{DISCUSSION}

Growth of the disease caused by the group A b hemolytic streptococci is the most brightly observed from the middle of 
Table 6. Results of Rapid Antigen Detection Test (RADT) and Bacterial Culture Analysis (BA)

\begin{tabular}{|l|r|}
\hline \multicolumn{1}{|c|}{$\mathbf{n}$} & \multicolumn{1}{|c|}{ Frequency (\%) } \\
\hline \hline \multicolumn{2}{|c|}{ GABHS positive by RADT } \\
\hline Total (n=200) & $72(36.0 \%)$ \\
\hline Positive by BA (n=80) & $54(67.5 \%)$ \\
\hline Negative by BA (n=120) & $18(15.0 \%)$ \\
\hline GABHS negative by RADT & \\
\hline Total (n=200) & $128(64.0 \%)$ \\
\hline Positive by BA (n=80) & $26(32.5 \%)$ \\
\hline Negative by BA (n=120) & $102(85.0 \%)$ \\
\hline GABHS: Group A $\beta$-hemolytic streptococcus.
\end{tabular}

80th of century XX in many countries of the world [17-20]. Thus, the prevalence of diseases caused by GABHS keeps the actual danger not only in developing countries, but also in developed countries.

In this study we demonstrate the high prevalence of GABHS among 200 children with chronic tonsillopharyngitis, detected by RADT and by culture method (Table 3), which requires a high attention. Thus, the study used a high-sensitive rapid antigen test (QuickVue Dipstick Strep A test) with a high specificity (100\%) and sensitivity $(94.0 \%)$ [21], but in other data high specificity $(99.4 \%)$ and low sensitivity (65.6\%) [22]; and analysis of data from the bacterial culture allowed us to determine the cause of growth of RF in an isolated region. Bacteria culture analysis has become the universally accepted "gold" standard for diagnostics of tonsillopharyngitis of the streptococcal etiology. In this our study RADT showed that its specificity equals to $85 \%$ and sensitivity is $67.5 \%$ (Table 6). Therefore, our attention is more accented on the results received by the culture method. $72(36.0 \%)$ positive children by RADT and $80(40.0 \%)$ positive children by BA for GABHS in total from all 200 subjects and 24 (47.0\%) positive children by BA out of 51 children with RF, despite of spent therapy by antibiotic, it is a very serious data. As we have noticed earlier (please see the Study material), at the moment of the study many children received antibacterial therapy, and it was not criterion of exception. It is a fact, that in many cases the pathogenic streptococcus remains absolutely resistant to used antibiotics. Besides, in this study we have tried to show the prevalence of GABHS in different ethnic groups: Kyrgyz and Russian (Tables $\mathbf{4}$ and 5). Unfortunately, we could not find more than 12 Russian subjects in that study timeframe, who met necessary requirements of the research. Most number of Kyrgyz children with positive results for GABHS: 18 (36.7\%) by RADT and $24(48.9 \%)$ by BA out of 51 total children with RF (Table 4), 51 (36.6\%) by RADT and $54(38.8 \%)$ by BA out of total children without RF (Table 5) testifies about predisposition to the high sensitivity and as a consequence to a large prevalence of this microbe among this ethnic population. The dramatic growth of the streptococcal tonsillopharyngitis and its complications, such as RF/RHD should be observed among the Kyrgyz population in
Kyrgyzstan [11]. Furthermore, prevalence of GABHS in female group was higher than in male group (Fig. 1). Children with RF from female group had $50 \%$ positive results by the culture method in compare with children with RF from male group $(41.1 \%)$ and children without RF from female group had $45.4 \%$ positive results by the culture method in compare with children without RF from male group (29.1\%). According to these derived data, we can not exclude that females are more sensitive and are more exposed to action of this pathogenic microbe. Consequently, females are frequent carriers and wide spreaders of a streptococcal infection in this country. More exact answer to this question can be received only after a wide randomized study of the population of Kyrgyzstan.

It is interesting to analyze the prevalence of GABHS in various age groups (Fig. 2). Prevalence of GABHS, received by culture method, from the younger age group (3-6 years) among children with RF showed $57.1 \%$, in group 7-12 years was $50 \%$, and in group $13-17$ years was $40.9 \%$ that probably presents a low sensitivity to used antibiotics and irregular secondary prophylaxis. Moreover, the results suggest that the prevalence and colonization of the oropharynx in children without RF by this bacterium is higher in last group (13-17 years) where were $41.4 \%$ positive results by BA, compared to the groups 3- 6 years and 7-12 years. The results of this study also suggest that the school children may represent a risk factor for colonization of oropharynx by the Streptococcus pyogenes. The GABHS transmission can be done through immediate contact, by respiratory tract and through food, facilitated by the gathering observed in schools. As you have noted from this study, the number of children with tonsillopharyngitis attending nursery schools are not so many; it can be justifiable by the fact that many kindergartens do not function in Kyrgyzstan after the USSR breakdown and economic declines in this country, so many younger children stay at home.

In addition to the detecting of GABHS, we have discovered some pathogenic microorganisms from oropharynx during conducting of our study (Fig. 3A). A specific value has a detection of the pathogenic bacteria as $\mathrm{S}$. pneumoniae $(18 \%)$ and beta-lactamase producing bacteria as S. aureus $(13 \%)$ that complicates treatment by antibiotics. The school children with chronic or persistent tonsillopharyngitis are a risk factor of colonization of oropharynx by the various pathogenic microorganisms.

All discovered GABHS was studied for susceptibility to antibiotics (Fig. 3B). Only $12.5 \%$ of GABHS positive samples out of total 80 samples were sensitive to penicillin. This pathogenic microbe was more sensitive to ampicillin $36.2 \%$ and to ceftriaxon $38.7 \%$ (cephalosporin). The most sensitive Streptococcus pyogenes has appeared to amoxicillin $45.0 \%$. Low sensitivity has appeared to macrolids: roxitromycin $26.2 \%$ and erythromycin $23.7 \%$. In time of the USSR the empirical prescription of antibiotics was based on knowledge of natural sensitivity of bacteria, epidemiological data of resistance of microorganisms in region or a hospital, and also on results of controllable clinical researches. Unfortunately, randomized researches have not been conducted in Kyrgyzstan during the last 15 years. It should be noted that $26.2 \%$ of GABHS positive samples in this study were absolutely resistant for all these 
antibiotics. It is necessary to carry out the wide clinical and microbiological studies. Data of results of definition of susceptibility to antibiotics can serve as a good reference point to clinical physicians for a choice and correction of antibacterial therapy. Considering the recognized streptococcal etiology of rheumatic fever at present, the penicillin is the drug of choice for eradication of GABHS in its initial clinical displays and for prevention of recurrence of infection. It has been established, that the treatment by phenoxymethylpenicillin during 10 days is optimum for achievement and full eradication of GABHS from throat [23, 24]. Besides the clinical and bacteriological efficiency is well documentary for amoxicillin, benzathine benzylpenicillin, amoxicillin/clavulanate, oral cephalosporin [25-33]. In comparative randomized research it has been shown, that prescription of amoxicillin/clavulanate potassium allows to amount to $100 \%$ eradication of GABHS in comparison with $70 \%$ eradication at use of penicillin $(p<0,001)$ [34]. In cases of allergy to penicillin, macrolide or cephalosporin is recommended. Also in a number of clinical tests high clinical and bacteriological efficiency of microlids - clarithromycin, roxithromycin, spiramycin and others is confirmed [35-37].

Thus, in the summary, we would like to emphasize once more that the basic reasons of increase prevalence of RF/RHD in Kyrgyzstan maybe due to the high prevalence of GABHS among school children, inefficiency and irregularity primary and secondary preventive measures.

\section{ACKNOWLEDGEMENTS}

This study was supported in part by National Institute of Biomedical Innovation, the Japanese Ministry of Education, Culture, Sports, Science and Technology, the Japanese Ministry of Health, Labour and Welfare, the Kato Memorial Trust for Nanbyo Research, the Japan Medical Association, Nagao Memorial Fund, Kanae Foundation for Life \& Sociomedical Science, Japan Research Foundation for Clinical Pharmacology, Kanagawa Nanbyo Foundation, Kanagawa Academy of Science, Technology Research Grants, Japan College of Rheumatology, the Nakajima Foundation, Osaka Foundation for Cancer Research Japan Society for the Promotion of Science, The Mishima Kaiun Memorial Foundation, the CALPIS Co., Ltd, Mitsui Life Insurance Co., Ltd and the Uehara Memorial Foundation. and Takeda Science Foundation, Heiwa Nakajima Foundation, The Sagawa Foundation for Promotion of Cancer Research, The Tokyo biochemical Research Foundation, The Kanagawa High-Technology Foundation and The Organization of Future of Kids in Silk Road.

\section{REFERENCES}

[1] Omurzakova NA, Yoshihisa Y, Saatova GM, et al. High incidence of rheumatic fever and rheumatic heart disease in republics of Central Asia. Int J Rheum Dis 2009; 12: 79-83.

[2] Carapetis JR, Steer AC, Mulholland EK, et al. The global burden of group A streptococcal diseases. Lancet Infect Dis 2005; 5: 68594.

[3] Atlas of Heart Diseases and Stroke. Available from URL: http://www.who.int/cardivascular_diseases/resources/atlas/en/Rheu matic fever and rheumatic heart disease 2004.

[4] Rheumatic fever and RHD. Report of a WHO Expert Consultation. Geneva 2001.

[5] Jonathan R. Carapetis rheumatic heart disease in developing countries. N Engl J Med 2007; 357: 439-41.
[6] Rheumatic fever and rheumatic heart disease: report of a WHO expert consultation. World Health Organ Tech Rep Ser 2004; 923: $1-122$.

[7] Kawakita S. Rheumatic fever and rheumatic heart disease in Japan. Jpn Circ J 1986; 50 (12): 1241-45.

[8] Brook I, Dohar JE. Management of group A beta-hemolytic streptococcal pharyngotonsillitis in children. J Fam Pract 2006; 55(12): S1-11; quiz S12.

[9] Pichicheri ME. Group A streptococcal tonsillopharyngitis: costeffective diagnosis and treatament. Ann Emerg Med 1995; 25: 390403.

[10] Vieira FM, Figueiredo CR, Soares MC, et al. Prevalence of Streptococcus pyogenes as an oropharynx colonizer in children attending daycare: a comparative study of different regions in Brazil. Rev Bras Otorrinolaringol 2006; 72(5): 587-91.

[11] Omurzakova NA, Yoshihisa Y, Tomoo S, et al. Increased prevalence of group A b-hemolytic streptococcus among an ethnic population in Kyrgyzstan detected by the rapid antigen detection test. Mol Med Report 2008; 1: 869-74.

[12] Bisno AL, Gerber MA, Gwaltney JM, Kaplan EL, Schwartz RH. Infectious Diseases Society of America. Practice guidelines for the diagnosis and management of group A streptococcal pharyngitis. Clin Infect Dis 2002; 15: 113-25.

[13] WHO. Basic laboratory procedures in clinical bacteriology. Geneva: WHO 1991.

[14] WHO. Basic laboratory procedures in clinical bacteriology. $2^{\text {nd }} \mathrm{ed}$. Geneva: WHO 2003.

[15] National committee on clinical laboratory standards. performance standards for antimicrobial susceptibility testing. Ninth Informational Supplement M100-S9. USA: NCCLS 1999; vol. 19: p. N1.

[16] Clinical laboratory standards institute: performance standards for antimicrobial susceptibility testing: seventh informational supplement M100-S15. Pennsylvania, USA; CLSI, Wayne 2005.

[17] Bisno AL, Brito MO, Collins CM. Molecular basis of group A streptococcal virulence. Lancet Infect Dis 2003; 3: 191-200.

[18] Allan G, John BZ. Rheumatic fever: etiology, diagnosis, and treatment. // Arthritis and allied conditions. A textbook of Rheumatology $14^{\text {th }}$ Edition. William J. Koopman. Volume 2. 2001; 1486: 1761-73.

[19] Schwartz B, Facklam RR, Breiman RF. Changing epidemiology of streptococcal infection in the USA. Lancet 1990; 336: 1167-71.

[20] Kaplan EL. Global assessment of rheumatic fever and rheumatic heart disease at the clouse of the century. influences and dynamics of populations and pathogens: a failure to realize prevention? Circulation 1993; 88: 1964-72.

[21] Kawakami S, Ono Y, Yanagawa Y, Miyazawa Y. Basic and clinical evaluation of the new rapid diagnostic kit for detecting group A streptococci with the immunochromatographical method. Rinsho Biseibutshu Jinsoku Shindan Kenkyukai Shi 2003; 14(1): 916.

[22] Van Limbergen J, Kalima P, Taheri S, Beattie TF. Streptococcus A in pediatric accident and emergency: are rapid streptococcal tests and clinical examination of any help? Emerg Med J 2006; 23(1): 32-34.

[23] Schwartz RH, Wientzen RL, Pedreira F, et al. Penicillin V for Group A streptococcal pharyngotonsillitis. A randomized triel of seven vs ten days'therapy. JAMA 1981; 246: 1790-95.

[24] Gerber MA, Randolph MF, Chanatry J, et al. Five days versus ten days of penicillin therapy for streptococcal pharyngitis. Am J Dis Child 1987; 141: 224-27.

[25] Cohen R, Levy K, Doit C, et al. Six-day amoxicillin vs ten day penicillin $\mathrm{V}$ therapy for group A streptococcal tonsillopharyngitis. Pediatr Infect Dis J 1996; 15 (8): 678-82.

[26] Lan AJ, Colford JM. The impact of dosing frequency in the efficacy of 10-day penicillin or amoxicillin therapy for streptococcal tonsillopharyngitis: a meta-analysis. Pediatrics 2000; 105 (2): E191-E201.

[27] Randolph MF. Clinical comparison of once-daily cefadroxil and thrice-daily cefaclor in the treatment of streptococcal pharyngitis. Chemotherapy 1988; 34: 512-18.

[28] Gerber MA, Randolph MF, Chanatry J, et al. Once-daily therapy for streptococcal pharyngitis with cefadroxil. J Pediatr 1986; 109: S31-S37. 
[29] Bingen E, Fitoussi F, Doit C, Cohen R et al. Resistance to macrolides in Streptococcus pyogenes in France in pediatric patients. Antimicrob Agents Chemother 2000; 44(6): 1453-57.

[30] Holm SE, Roos K, Stromberg A. A randomised study of treatment of streptococcal pharyngotonsillitis with cefadroxil or phenoxymethylpenicillin (penicillin V). Pediatr Infect Dis J 1991; 10: S68-71.

[31] Shvartzman P, Tabenkin H, Rosentzwaig A, et al. Treatment of streptococcal pharyngitis with amoxicillin once a day. Br Med J 1993; 306(6886): 1170-3.

[32] Dykhuizen RS, Golder D, Reid TMS, et al. Phenoxymethylpenicillin versus co-amoxiclav in treatment of acute streptococcal pharyngitis and the role of beta-lactamase activity in saliva. J Antimicrob Chemother 1996; 37(1): 133-8.

[33] Peyramond D, Portier H, Geslin P, Cohen R. 6 - day amoxicillin versus 10 - day penicillin $\mathrm{V}$ for group A B-haemolitic streptococcal acute tonsillitis in adults: A French multicentre, open - label, randomized study. Scand J Infect Dis 1996; 28(5): 497-01.

[34] Brook I. Treatment of patients with acute recurrent tonsillitis due to group A beta-haemolytic Streptococci a prospective randomized study comparing penicillin and amoxicillin/clavulanate potassium J Antimicrob Chemother 1989; 24(2): 227-33.

[35] Bachand RTJr. A comparative study of clarithromycin and penicillin VK in the treatment of outpatients with streptococcal pharyngitis. J Antimicrob Chemother 1991; 27 (Suppl A): 75-82.

[36] Kaplan EL, Gooch WM III, Notario GF, Craft JC. Macrolide therapy of group A streptococcal pharyngitis: 10 days of macrolide therapy (clarithromycin) is more effective than 5 days (azithromycin). Clin Infect Dis 2001; 32: 1798-02.

[37] Gendrel D, Bourrillon A, Bingen E, et al. Five-day spiramycin vs seven-day penicillin $\mathrm{V}$ in the treatment of streptococcal tonsillitis in children. Clin Drug Invest 1997; 13(6): 338-44.

Received: May 25, 2010

Revised: July 5, 2010

Accepted: July 16, 2010

(c) Omurzakova et al.; Licensee Bentham Open.

This is an open access article licensed under the terms of the Creative Commons Attribution Non-Commercial License (http://creativecommons.org/licenses/by-nc/ 3.0/) which permits unrestricted, non-commercial use, distribution and reproduction in any medium, provided the work is properly cited. 